\title{
Communication
}

[Comunicação]

\section{Isolation of Salmonella spp. in captive Psittaciformes from zoos and a commercial establishment of Fortaleza, Brazil}

\author{
[Isolamento de Salmonella spp. em Psittaciformes de zoológicos e estabelecimento \\ comercial de Fortaleza, Brasil]
}

E.S. Lopes, W.M. Cardoso*, Á.H. Albuquerque, R.S.C. Teixeira, R.P.R. Salles, W.G. A. Bezerra, R.C. Rocha e Silva, S.V.G. Lima, R.J.P.F. Sales, R.H.Vasconcelos

Universidade Estadual do Ceará - UECE - Fortaleza, CE

Studies have emphasized the importance of wild birds in the transmission of pathogenic microorganisms to other animals, whether wild or industrial birds, as well as in humans. Among these microorganisms, Salmonella spp. has shown relevant importance. Sousa et al. (2010a) captured 48 wild birds near poultry facilities in the North of São Paulo city, Brazil and detected three birds positive for Salmonella (Zenaida auriculata, Cariama cristata and Theristicus caudatus), which demonstrated that free-living birds may carry and disseminate pathogenic agents, presenting a risk for poultry. Silva et al. (2010) emphasized the importance of members of the Ciconiiformes order as carriers of Salmonella to other wild birds as well as to poultry.

Another group of free-living birds that have demonstrated a potential role as reservoirs of Salmonella are the Psittaciformes, since this microorganism have already been isolated from psittacines in their natural environment (Vilela et al., 2001; Allgayer et al., 2009). Therefore, the possibility of transmission of this pathogen to other free-living or to domestic birds cannot be unconsidered. However, due to the low number of studies currently performed concerning this topic and to the fact that there is no evidence that psittacines are a major source of Salmonella to domestic fowl, the psittacines are not included among the free-living birds that cause concern to the poultry industry in this aspect (Butron and Brightsmith, 2010; Sousa et al., 2010a).

Most studies relating Salmonella to psittacines involve birds maintained in captivity. The maintenance conditions of captive birds favor the spread of infectious diseases (Mattes et al., 2005), which may affect negatively not only the health of other birds, but of human beings as well. Several diseases are transmitted from captive or pet birds to humans through direct or indirect contact with sick or asymptomatic birds, and bacteria are the most common zoonotic agents (Akhter et al., 2010). From the reports about several serotypes of Salmonella isolated from wild birds come the importance of these microorganisms in the epidemiology of human and animal salmonellosis (Hughes et al., 2008). In Brazil, although few studies have been published in the last decade addressing the presence of Salmonella spp. in Psittaciformes, they are still scarce, which makes further research necessary in order to understand the serotypes capable of infecting birds in captivity. Therefore, this study aimed to investigate the frequency of Salmonella spp. with the aid of cloacal swabs in captive psittacines from commercial and conservational facilities of the city of Fortaleza, Brazil.

One hundred and eighty-two members of the Psittaciformes order corresponding to a total of 36 species (Table 1), especially parakeets, parrots and macaws from two zoos and a commercial establishment were analyzed. At the sampling moment, all birds were apparently healthy, therefore with no clinical signs of salmonellosis. The bacterial isolation was performed as described by Sousa et al. (2010b). Isolates identified as Salmonella spp. were streaked on nutrient agar (Oxoid $\AA$ ) and sent to complete identification and serotyping carried out by the Fundação Oswaldo Cruz in Rio de Janeiro, Brazil. This study was approved by the Ethics Committee for the Use of Animals with the following number 10339107-0/48.

Recebido em 18 de março de 2013

Aceito em 18 de dezembro de 2013

* Autor para correspondência (corresponding author)

E-mail: william.maciel@uol.com.br 
Lopes et al.

Table 1. Psittaciforme species, common name, positive sample/number examined and Salmonella isolated from captive psittacine

\begin{tabular}{|c|c|c|c|}
\hline Species & Common Name & (n) & Positive Samples \\
\hline Amazona aestiva & Blue-fronted Amazon & $1 / 31$ & Salmonella Lexington \\
\hline Amazona festiva & Festive Amazon & $0 / 02$ & - \\
\hline Amazona ochrocephala & Yellow-crowned Amazon & $0 / 03$ & - \\
\hline Amazona farinosa & Mealy Amazon & 0/07 & - \\
\hline Amazona amazônica & Orange-winged Amazon & $0 / 06$ & - \\
\hline Alipiopsitta xanthops & Yellow-faced Parrot & $0 / 04$ & - \\
\hline Deroptyus accipitrinus & Red-fan Parrot & $0 / 01$ & - \\
\hline Amazona rhodocorytha & Red-browed Amazon & $0 / 04$ & - \\
\hline Ara ararauna & Blue-and-yellow Macaw & $0 / 12$ & - \\
\hline Ara macao & Scarlet Macaw & $0 / 11$ & - \\
\hline Ara chloroptera & Red-and-green Macaw & $1 / 16$ & Salmonella Saintpaul \\
\hline Anodorhynchus hyacinthinus & Hyacinth Macaw & $0 / 04$ & - \\
\hline Aratinga cactorum & Caatinga Parakeet & $0 / 06$ & - \\
\hline Aratinga leucophthalma & White-eyed Parakeet & $0 / 10$ & - \\
\hline Aratinga auricapillus & Golden-capped Parakeet & $0 / 03$ & - \\
\hline Aratinga jandaya & Jandaya Parakeet & $0 / 07$ & - \\
\hline Aratinga solstitialis & Sun Parakeet & $0 / 03$ & - \\
\hline Aratinga aurea & Peach-fronted Parakeet & $0 / 02$ & - \\
\hline Guaruba guarouba & Golden Parakeet & $0 / 02$ & - \\
\hline Pyrrhura perlata & Crimson-bellied Parakeet & $0 / 03$ & - \\
\hline Pyrrhura griseipectus & Gray-breasted Parakeet & $0 / 03$ & - \\
\hline Pionus fuscus & Dusky Parrot & $0 / 01$ & - \\
\hline Pionus menstruus & Blue-headed Parrot & $0 / 13$ & - \\
\hline Nandayus nenday & Nanday Parakeet & $0 / 01$ & - \\
\hline Pionitis leucogaster & White-bellied Parrot & $0 / 03$ & - \\
\hline Pionites melanocephalus & Black-headed Parrot & $0 / 01$ & - \\
\hline Ara severus & Chestnut-fronted Macaw & $0 / 02$ & - \\
\hline Ara maracanã & Blue-winged Macaw & $0 / 03$ & - \\
\hline Primolius auricollis & Golden-collared Macaw & $0 / 02$ & - \\
\hline Gradidascalus brachyurus & Short-tailed Parrot & $0 / 03$ & - \\
\hline Brotogeris chiriri & Yellow-chevroned Parakeet & $0 / 01$ & - \\
\hline Nymphicus hollandicus & Cockatiel & $0 / 03$ & - \\
\hline Eclectus roratus & Eclectus Parrot & $0 / 01$ & - \\
\hline Psittacula krameri & Ringnecked Parakeet & $0 / 01$ & - \\
\hline Cacatua galerita galerita & Sulphur-crested Cockatoo & $0 / 01$ & - \\
\hline Melopsittacus undulatus & Budgerigar & $1 / 06$ & Salmonella Newport \\
\hline Total & - & $03 / 182$ & - \\
\hline
\end{tabular}

A prevalence of $1.65 \%$ was found from a total of 182 psittacines analyzed with the following serotypes Lexington, Saintpaul and Newport isolated from Amazona aestiva, Ara chloroptera and Melopsittacus undulatus, respectively. This appears to be the first reported isolation of these serotypes from these studied psittacine species.

In Brazil, Marietto-Gonçalves et al. (2010a) investigated 89 cloacal swab samples from three different psittacine species and a single Salmonella sp. strain was isolated from an
Amazona aestiva, representing $1.12 \%$ from the total analyzed. The same authors isolated the serotype Enteritidis from Amazona aestiva originated from wild fauna traffic in São Paulo, Brazil (Marietto-Gonçalves et al., 2010b). Oliveira et al. (2009) detected Salmonella Hadar in 49 parrots (Amazona aestiva and Amazona amazona) (8.2\%) aging from one to six-monthold. Akhter et al. (2010) found Salmonella spp. in 45 samples of cloacal, oral and fecal swabs from psittacines allocated at the Dhaka zoo in Bangladesh reporting a prevalence of $46.67 \%$. In 
captive psittacines, several authors described the isolation of various serotypes of Salmonella spp., but Typhimurium seems to be the most frequent (Allgayer et al., 2008). This is demonstrated as well by Piccirillo et al. (2010) who isolated the serotype Typhimurium DT 160 from Cacatua moluccensis in a zoo in Italy. This serotype may manifest as a primary pathogen, or a subclinical infection, in young or weak birds (Vigo et al., 2009).

This study showed a low prevalence of Salmonella spp. in captive psittacines from zoos and a commercial establishment of Fortaleza. None of the isolated serotypes ( $S$. Lexington, $S$. Saintpaul and $S$. Newport) have yet been reported in Amazona aestiva, Ara chloroptera or Melopsittacus undulatus. However, the fact that most birds presented negative for Salmonella spp. may not imply the absence of this pathogen in these birds, since the intermittent excretion is a well-known characteristic of this microorganism.

Keywords: Salmonella, Psittaciformes, Amazona aestiva, Ara chloroptera, Melopsittacus undulatus

\section{RESUMO}

A manutenção de aves em cativeiro reúne condições que favorecem a disseminação de doenças infecciosas, sendo a Salmonella uma dessas doenças infecciosas que acomete os psitacídeos. Portanto o objetivo do presente estudo foi isolar e identificar Salmonella spp. em Psittaciformes mantidos em criatórios comerciais e conservacionistas da Região Metropolitana de Fortaleza. Para o estudo, foram coletados swabs cloacais de 182 psitacídeos clinicamente sadios. Os resultados mostraram que três psitacídeos avaliados (1,65\%) foram positivos: Amazona aestiva (Salmonella Lexington), Ara chloroptera (Salmonella Saintpaul) e Melopsittacus undulatus (Salmonella Newport). De acordo com a literatura científica, não há registro desses sorotipos em psitacídeos. Esta pesquisa evidenciou uma baixa prevalência de Salmonella spp. em Psittaciformes mantidos em criatórios comerciais e conservacionistas da Região Metropolitana de Fortaleza.

Palavras-chave: Salmonella, Psittaciformes, Amazona aestiva, Ara chloroptera, Melopsittacus undulatus

\section{ACKNOWLEDGMENTS}

We acknowledge the Conselho Nacional de Desenvolvimento Científico e Tecnológico (CNPq) and the Laboratório de Estudos Ornitológicos (LABEO/FAVET/UECE) for their support to this study.

\section{REFERENCES}

AKHTER, J.M.; HOSSAIN.; ISLAM, M.T. et al. Isolation and identification of microflora from apparently healthy caged parrots of Dhaka Zoo of Bangladesh. Bangl. J. Vet. Med., v.8, p.5-10, 2010.

ALLGAYER, M.C.; LIMA-ROSA, C.A.; WEIMER, T.A. et al. Molecular diagnosis of Salmonella species in captive psittacine birds. Vet Rec., v.162, p.816-819, 2008.

ALLGAYER, M.C.; OLIVEIRA, S.J.; MOTTIN, V.D. et al. Isolamento de Salmonella Braenderup em arara-azul (Anodorhynchus hyacinthinus). Cienc. Rural, v.39, p.2542-2545, 2009.
BUTRON, O.; BRIGHTSMITH, D.J. Testing for Salmonella spp. in released parrots, wild parrots, and domestic fowl in lowland Peru. J. Wildl. Dis., v.46, p.718-723, 2010.

HUGHES, L.A.; SHOPLAND, S.; WIGLEY, P. et al. Characterization of Salmonella enterica serotype Typhimurium isolates from wild birds in northern England from 2005 - 2006. BMC. Vet. Res., v.4, p.1-10, 2008.

MARIETTO, G.G.A.; ALMEIDA S.M.; LIMA, E.T.; FILHO, R.L.A. Detecção de Escherichia coli e Salmonella sp. em microbiota intestinal de Psittaciformes em fase de reabilitação para soltura. Braz. J. Vet. Res. Anim. Sci., v.47, p.185189, 2010a.

MARIETTO, G.G.A.; ALMEIDA S.M. et al. Isolation of Salmonella enterica Serovar Enteritidis in Blue-Fronted Amazon Parrot (Amazona aestiva). Avian Dis., v.54, p.151-155, 2010b. 
MATTES, B.R.; CONSIGLIO, S.A.S.; ALMEIDA, B.Z. et al. Influência da biossegurança na colonização intestinal por Escherichia coli em psitacídeos. Arq. Ins. Biol., v.72, p.13-16, 2005.

OLIVEIRA, C.Z.F.; BOAKARI, Y.L.; BRAGA, J.F.V.; NOBRE, M.L.M. Detecção de Salmonella sp. em papagaios (A. aestiva e A. amazona) de vida livre apreendidos no Estado do Piauí. In: XXV CONGRESSO BRASILEIRO DE MICROBIOLOGIA, 2., 2009, Porto de Galinhas, PE. Anais... Microbiologia In foco. São Paulo: [s.n] 2009 p. 108. (Resumo).

PICCIRILLO, A.; MAZZARIOL, S.; CALIARI, D.; MENANDRO M.L. Salmonella Typhimurium Phage Type DT160 Infection in Two Moluccan Cockatoos (Cacatua moluccensis): Clinical Presentation and Pathology. Avian Dis., v.54, p.131-135, 2010.

SILVA M.A.; MARVULO M.F.V.; MOTA R.A.; SILVA J.C.R. A importância da ordem Ciconiiformes na cadeia epidemiológica de Salmonella spp. para a saúde pública e a conservação da diversidade biológica. Pesq. Vet. Bras., v.30, p.573-580, 2010.
SOUSA E.; WERTHER K.; BERCHIERI JÚNIOR A. Assessment of Newcastle and infectious bronchitis pathogens, and Salmonella spp. in wild birds captured near poultry facilities. Arq. Bras. Med. Vet. Zootec., v.62, p.219-223, 2010a.

SOUSA, E.; BERCHIERI, A.J.; PINTO, A.A. et al. Prevalence of Salmonella spp. antibodies to Toxoplasma gondii, and Newcastle disease vírus in feral pigeons (Columba livia) in the city of Jaboticabal, Brazil. J. Zoo. Wildlife Med., v.41, p.603-607, 2010b.

VIGO, G.B.; ORIGLIA, J.; GORNATTI, D. et al. Isolation of Salmonella Typhimurium from dead blue and gold macaws (Ara ararauna). Avian Dis., v.53, p.135-138, 2009.

VILELA, V.O.; GUEDES, N.M.R.; ARAÚJO, F.R. et al. Salmonella Bredeney em Arara-Azul (Anodorhynchus hyacinthinus). In: CONGRESSO BRASILEIRO DE ORNITOLOGIA. 9., 2001, Curitiba-Paraná. Ornitologia sem fronteiras. Curitiba: Fernando Costa Straube, 2001. p.390391. 